\title{
Scroll waves meandering in a model of an excitable medium
}

\author{
A. Rusakov and A. B. Medvinsky \\ Institute for Theoretical \& Experimental Biophysics, Russian Academy of Sciences Pushchino, Moscow Region 142290, Russia
}

A. V. Panfilov

Theoretical Biology, Utrecht University, Padualaan 8, Utrecht 3584 CH, The Netherlands

(Received 19 April 2005; revised manuscript received 14 June 2005; published 25 August 2005)

\begin{abstract}
We study numerically the dynamics of a scroll wave in a three-dimensional (3D) excitable medium in the presence of substantial meandering of the corresponding 2D spiral wave in the Aliev-Panfilov model. We identify three types of dynamics of the scroll wave filament-quasi-2D, periodic, and aperiodic meanderingand we study their dependence on parameter settings and thickness of the medium.
\end{abstract}

DOI: 10.1103/PhysRevE.72.022902

PACS number(s): 87.19.Hh, 87.19.Nn, 87.18.Pj, 82.40.Np

Scroll waves are three-dimensional (3D) vortices which are extensions of the well-known spiral waves that occur in a variety of excitable media. Scroll waves have been observed in the Belousov-Zhabotinski (BZ) chemical reaction [1,2], in the slug phase of the life cycle of slime molds [3] and in the ventricles of the heart during cardiac arrhythmias [4,5]. Numerous modeling studies of scroll waves have been performed using analytical and numerical methods. The importance of scroll waves for our understanding of the behavior of excitable media has been consistently emphasized in the literature [6].

A scroll wave is usually characterized by its filament [7], which is an extension into three dimensions of the notion of the core of the spiral wave. In general, the dynamics of a filament in $3 \mathrm{D}$ is governed by its curvature, twist [8,9], and by the anisotropy of the excitable medium $[6,10,11]$. Mathematically, drifts occur as a result of a convective term in the Laplacian in a coordinate system with an axis directed along the filament and can be effectively studied using singular perturbation theory [9]. The curvature-induced drift of the filament changes the filament length. This property of the filament is often regarded as the filament tension [12], which can be positive or negative [8]. If the tension is negative, the filament increases in length, leading to instability, which can cause the multiplication of scrolls $[12,13]$. If the tension of the filament is positive, the filament tends to become shorter, which results either in the collapse of scroll waves with a closed (circular) filament or in the stabilization of a straight filament between two opposite boundaries in a uniform medium [14]. Filament behavior is highly important for the system in which scroll waves occur. For example, it is a widely accepted hypothesis that different orientations of filaments in the heart and different types of their dynamics determine the type of cardiac arrhythmia and its possible deterioration into fibrillation in the ventricles of the heart $[15,16]$.

Until recently, the dynamics of filaments was mainly studied for those parameters of an excitable medium that show stable rotation of a 2D spiral wave, i.e., for so-called circular cores. However, the assumption of a circular core does not hold for several important practical cases: spiral waves in a $\mathrm{BZ}$ reaction [17] and spiral waves in models of cardiac tissue
[18] both show pronounced meandering [19]. In this article, we study filament dynamics in the presence of scroll wave meandering in a model for cardiac tissue [20].

\section{MODEL AND METHODS OF COMPUTATION}

The excitable medium was described by the AlievPanfilov model [20],

$$
\begin{gathered}
\partial e / \partial t=\nabla^{2} e-k e(e-a)(e-1)-e r, \\
\partial r / \partial t=\left[\epsilon+\left(\mu_{1} r\right) /\left(\mu_{2}+e\right)\right][-r-k e(e-b-1)] .
\end{gathered}
$$

Here the variable $e$ stands for the transmembrane potential and variable $r$ represents the conductance of the slow inward current. The function $-k e(e-a)(e-1)$ in Eq. (1) determines the fast processes, such as the initiation of the upstroke of the action potential. The dynamics of the recovery phase of the action potential is determined by the time course of the variable $r$, mainly by the function $\left[\epsilon+\left(\mu_{1} r\right) /\left(\mu_{2}+e\right)\right]$. The particular parameters in this model do not have a clear physiological meaning but are adjusted to reproduce the key characteristics of cardiac tissue, such as the shape of the action potential, refractoriness, and the restitution of action potential duration. The values of the parameters used in this investigation are $\mu_{2}=1.3, k=8, \epsilon=0.01, b=0.1, \mu_{1}=0.2$; parameter $a$ was varied between 0.12 and 0.18 . Decreasing of $a$ increases the excitability of the cell and prolongs the duration of the action potential and the refractory period.

For numerical computations we used the explicit Euler method with Neumann boundary conditions, and a rectangular grid containing up to $128 \times 128 \times 128$ elements. To initiate the first scroll, we used initial data corresponding to a 3D broken wave front. Numerical integration was performed with a space step $h s=0.6$ and a time step $h t=0.03$ [21]. The dimensioned values of the time and space steps in this case were calculated on the basis of the properties of spiral waves in cardiac tissue [20] in our case these were $h t=0.077 \mathrm{~ms}$, $h z=0.1 \mathrm{~mm}$, and $h x=h y=0.3 \mathrm{~mm}$. The spiral wave core and the scroll wave filament were traced using an algorithm proposed by [22], by detecting the tip points as the intersection 


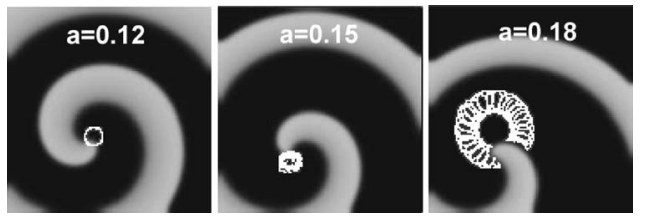

FIG. 1. Spiral wave and its core (the white lines) for $a=0.12$, $a=0.15$, and $a=0.18$. Computations were performed in a $2 \mathrm{D}$ excitable medium consisting of $128 \times 128$ elements. The light gray area represents the excited state of the tissue $(e>0.6)$ and intermediate shading from gray to black shows different levels of recovery.

point of an isopotential line (in our case 0.5) and the $d V / d t=0$ line.

\section{RESULTS}

Figure 1 shows the evolution of meandering patterns of 2D spiral waves for different values of the parameter $a$ of model (1) and (2). We see that for $a=0.12$ we have an almost circular core, for $a=0.15$ the spiral tip starts to meander slightly, and for $a=0.18$ the spiral wave meanders substantially.

We studied the dynamics of a scroll wave for the same parameter values, by extending our computations from the $128 \times 128$ plane to the $3 \mathrm{D}$ domain. In the first series of computations, we used the initial conditions translationally invariant in the third $(Z)$ direction. For that we copied the same 2D spiral wave pattern to all layers of our numerical grid. The meandering patterns in all $Z$ sections of the medium in this case were the same as in Fig. 1, and the filament remained a straight line with a length of $12.7 \mathrm{~mm}$ (thickness of the medium). Because of the similarity of this process with 2D meandering, we will call it quasi-2D meandering. In order to study the stability of this regime in $3 \mathrm{D}$, we performed a series of computations in which we shifted the whole 2D spiral wave for each $(z)$ slice of the system in the $x$ direction as $x_{\text {new }}=x_{\text {old }}+s * \sin [(\pi / 12.7) z]$, with $s=0.5 \mathrm{~mm}$ (estimated $5 \%$ disturbance of the filament length), and we studied whether the scroll wave filament returns to its unperturbed state with the minimal length. To represent this, we plotted the length of the filament in the course of time for the three different meandering regimes (Fig. 2). We found that for $a$

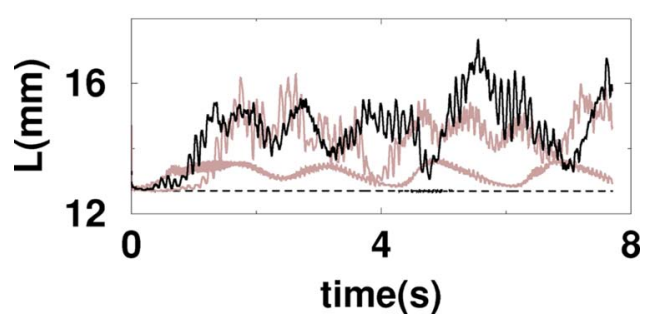

FIG. 2. (Color online) Filament length vs time. The parameter values are $a=0.18$ (black solid line), $a=0.18$ with the periodic boundary conditions (upper gray solid line), $a=0.15$ (lower gray solid line), and $a=0.12$ (long dashed line). Computations in the medium consist of $128 \times 128 \times 128$ elements with the initially sinusoidally perturbed straight filament with the Neumann boundary conditions (except the upper gray line). a

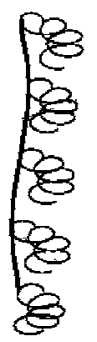

b

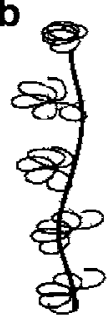

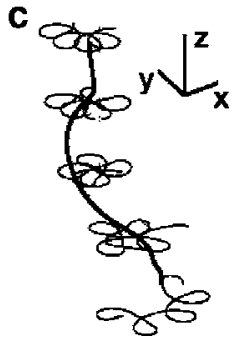

FIG. 3. Filament dynamics at $a=0.18$ at $t=0 \mathrm{~s}$ (the left), $t=1 \mathrm{~s}$ (the middle), and $t=3 \mathrm{~s}$ (the right) in the course of time.

$=0.12$ (corresponding 2D spiral has a circular core, Fig. 2 the dashed line), the filament length returns to the minimal length within $100 \mathrm{~ms}$, indicating that in this case we have a straight filament orthogonal to the top and the bottom boundaries of the domain. Thus in this case the quasi-2D meandering of the scroll wave is stable. However, even in the case of slight meander of the spiral wave $(a=0.15$, gray line), the filament length did not return to the thickness of the medium and after some transient period of time the filament length oscillated between the values of $L=12.8 \mathrm{~mm}$ and $L$ $=13.6 \mathrm{~mm}$. For strong meander of the spiral wave $(a=0.18$, solid line), we also see changes in the filament length, but they are more complex and have a larger amplitude (between $L=13.7 \mathrm{~mm}$ and $L=15.2 \mathrm{~mm}$ ). Note that, in all these cases the filament tension is positive: the computed effective diffusion coefficient $(D)$ relating filament curvature $(k)$ and contraction velocity $\left(V_{c}=D k\right)$ was $D=-0.29 \mathrm{~mm}^{2} / \mathrm{ms}$ for $a$ $=0.12, \quad D=-0.625 \mathrm{~mm}^{2} / \mathrm{ms}$ for $a=0.15$, and $D$ $=-0.841 \mathrm{~mm}^{2} / \mathrm{ms}$ for $a=0.18$. Furthermore, the effects of 3D filament meandering did not depend on the initial conditions. We performed simulations for initial disturbances with an amplitude of $s=0.3 \mathrm{~mm}$ and found qualitatively similar behaviors (not shown). We have also performed computations for the strong meandering case with periodic boundary conditions in the top and bottom of the medium (the upper gray line). We found that although the amplitude of the filament length oscillations was slightly less than for the Neumann boundary conditions, the complex aperiodic dynamics remained.

It can be seen that for $a=0.15$ and $a=0.18$, the dynamics of the filament length has two components: a high-frequency component with an amplitude of about $5 \%$ of the total filament length and frequency close to the average frequency of the spiral wave, and a low-frequency component on a much slower scale. The detailed patterns of filament meandering for $a=0.18$ are shown in Fig. 3. We see that initially the filament is only a slightly curved line (the left figure) and the meandering at each level is similar to the meandering of the 2D spiral wave in Fig. 1(c). However, in the course of time, the filament shape becomes more complex, and the meandering patterns at each level become different from each other substantially [Figs. 3(b) and 3(c)]. As a result, we obtain a complex aperiodic change of the filament length as shown in Fig. 2. We will discuss the dynamics of the filament and the nature of the fast and slow components after describing another series of computations for this case $(a=0.18)$ in which we varied the thickness of the medium. 


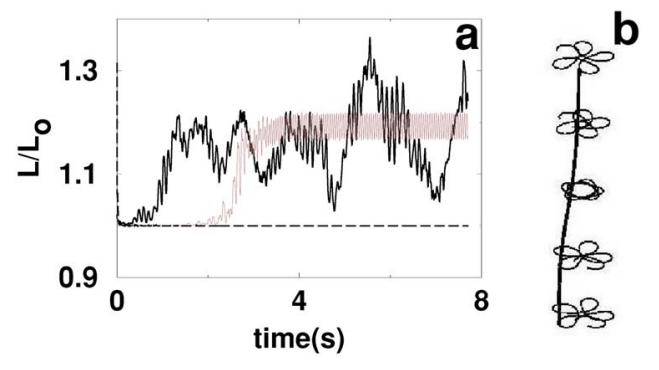

FIG. 4. (Color online) (a) Relative filament length vs time for $a=0.18$ in the medium of the thickness of $12.7 \mathrm{~mm}$ (the solid line), $3.1 \mathrm{~mm}$ (the gray line), and $2.5 \mathrm{~mm}$ (the dashed line). The relative filament length is defined as the filament length $(L)$ divided by the thickness of the medium $\left(L_{o}\right)$. (b) Filament meandering for the medium which is $3.1 \mathrm{~mm}$ thick.

We found that the 3D meandering pattern, in this case, crucially depends on medium thickness [Fig. 4(a)]. For small thickness $(2.5 \mathrm{~mm}$, dashed line $)$, the filament length $(L)$ approaches the thickness of the medium $\left(L_{o}\right)$, which indicates that the filament is straight and the meandering patterns in all $Z$ sections are the same. Thus in this case we have a quasi-2D meandering. For an intermediate thickness (3.1 mm, gray line) we see that the filament length increases by approximately $20 \%$, but meandering is periodic (the slow component of the filament length change is absent). For a thick excitable medium [solid line, Fig. 4(a)] we have the aperiodic meandering described earlier (Figs. 2 and 3). To represent these changes, we plotted the maximal, minimal, and average filament length of established meandering patterns for 14 different thicknesses of the medium [Fig. 5(a)]. We see that for medium thickness below $2.5 \mathrm{~mm}$, we have a quasi-2D meandering regime. For thicknesses between 2.6 and $3.2 \mathrm{~mm}$, we have periodic meandering, which is characterized by an increase in the average filament length and a relatively small difference between the minimal and maximal length. For thicknesses above $3.2 \mathrm{~mm}$, filament meandering becomes aperiodic, which is characterized by large variations of the filament length, however the average filament length does not differ much from that for the periodic meandering regime. Similar changes occur if we fix the thicknesses of the medium and increase the value of parameter $a$ [Fig. 5(b)]. We see the gradual change in the amplitude of filament meandering, which becomes more pronounced and aperiodic for $a \geqslant 0.15$.

The typical meandering pattern for the periodic regime is shown in Fig. 4(b). We see that the core patterns in the different sections do not change in the course of time, however
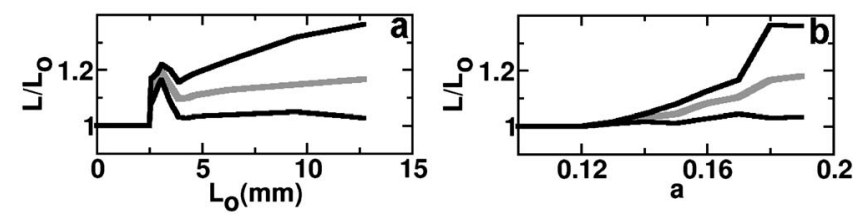

FIG. 5. (a) The maximal, minimal (solid lines), and the average (the gray line) relative filament length during the time from $4 \mathrm{~s}$ until $8 \mathrm{~s}$ vs thickness of excitable medium. (b) Same vs parameter $a$ for the medium which is $12.7 \mathrm{~mm}$ thick. the core shape depends substantially on the location of the section. As a result, we observe periodic changes in filament length that occur due to different rotation patterns of the scroll.

Now we can revisit the filament dynamics for the aperiodic regime shown in Fig. 3. The high-frequency component here, as in Fig. 4(b), is determined by the difference in the meandering patterns at different sections of the medium. However, there is another slow process on top of this periodic meandering. The result of this process is a substantial change of the filament shape. The increase in filament length usually starts as a bump, which occurs at a point around one-third of the way along the filament. This bump slowly shifts to the boundary of the medium and disappears there. As a result, the length of the filament decreases and reverts almost to its minimum value. In general, this slow dynamics is quite complex and shows substantial variations. For example, two bumps can develop on one filament or one bump can disappear and be replaced by another one, etc.

Figure 5 also suggests that the aperiodic filament dynamics occurs as a result of two subsequent (supercritical) bifurcations. Taking into account that the periodic meandering regime is a quasiperiodic motion, its bifurcation into an aperiodic regime may result in the onset of chaos via the quasiperiodic route [23]. However, due to computer resource limitations, we did not characterize how chaotic the pattern is during the aperiodic meandering.

\section{DISCUSSION}

In this paper we find three regimes of meandering of a scroll wave filament: quasi-2D, periodic, and aperiodic meandering. The periodic meandering is characterized by different meandering amplitudes at different cross sections of the medium and is similar to the meandering described in $[24,25]$. The aperiodic regime has more complex dynamics and is characterized by substantial buckling of the filament. The quasi-2D meandering is stable only in media of small thickness, or for a circular core of the corresponding 2D spiral waves. Note also that, as shown in [24,25], for some parameter values meandering in $3 \mathrm{D}$ can occur earlier than in 2D.

Filament instability was also studied numerically in another FitzHugh-Nagumo-type model [26]. It was shown that the meandering can result in instability whereby the filament acquires a helical shape. However, in that regime the average length of the filament monotonously increased with time and exceeded the minimum length by only $1 \%$, whereas in our case the average filament length was not monotonically increasing but instead showed substantial oscillations (of up to 20\%). The high-frequency component observed in [26] was similar to that observed in our paper.

Real cardiac tissue is nonuniformly anisotropic, and such an anisotropy can substantially affect the dynamics of scroll waves and cause the filament to acquire nonstraight shapes $[6,11]$. Note, however, that analysis and numerical modeling in $[6,11]$ were performed for the case of a circular core of spiral waves. Our results suggest that these stationary filament configurations may not hold for a meandering spiral where the filament can undergo complex motion. It is diffi- 
cult to say whether the same stable configurations will be found for the time-averaged filament position, since the approach proposed in $[6,11,27]$ may still work in the average sense, or that the asynchronous spiral meander and its interaction with rotational anisotropy will result in different final averaged configurations.

Although this work was carried out using a simplified model for cardiac tissue, we expect that the conclusions of this paper will be confirmed when more detailed models of cardiac tissue are used. Our expectation is based on the fact that spiral waves in ionic models of cardiac tissue meander substantially [18], which is likely to lead to 3D instabilities.
In conclusion, we found the following three dynamics of scroll waves in 3D excitable media: quasi-2D, periodic, and aperiodic meandering. These regimes occur in the presence of substantial meandering of the corresponding 2D spiral waves.

\section{ACKNOWLEDGMENTS}

We are thankful to Dr. McNab for linguistic advice and for helping to clarify the text, and Dr. K. H.W.J. ten Tusscher for a careful reading of the manuscript and valuable suggestions.
[1] A. T. Winfree, Science 181, 937 (1973).

[2] A. M. Pertsov, R. R. Aliev, and V. I. Krinsky, Nature (London) 345, 419 (1990).

[3] F. Siegert and C. J. Weijer, Proc. Natl. Acad. Sci. U.S.A. 89, 6433 (1992).

[4] A. M. Pertsov and J. Jalife, in Cardiac Electrophysiology. From Cell to Bedside, 2nd ed., edited by D. P. Zipes and J. Jalife (Saunders, Philadelphia, 1995), pp. 403-410.

[5] I. Efimov, V. Sidorov, Y. Cheng, and B. Wollenzier, J. Cardiovasc. Electrophysiol. 10, 1452 (1999).

[6] M. Wellner, O. Berenfeld, J. Jalife, and A. M. Pertsov, Proc. Natl. Acad. Sci. U.S.A. 99, 8015 (2002).

[7] A. T. Winfree and S. H. Strogatz, Nature (London) 311, 611 (1984).

[8] A. V. Panfilov and A. N. Rudenko, Physica D 28, 215 (1987).

[9] J. P. Keener and J. J. Tyson, SIAM Rev. 34, 1 (1992).

[10] F. H Fenton, S. J. Evans, and H. M. Hastings, Phys. Rev. Lett. 83, 3964 (1999).

[11] O. Berenfeld and A. M. Pertsov, J. Theor. Biol. 199, 383 (1999).

[12] V. N. Biktashev, A. V. Holden, and H. Zhang, Philos. Trans. R. Soc. London, Ser. A 347, 611 (1994).

[13] S. Alonso, F. Sagues, and A. Mikhailov, Science 299, 1722 (2003).
[14] A. V. Panfilov, R. Aliev, and A. Mushinsky, Physica D 36, 181 (1989); 36, 188 (1989).

[15] A. T. Winfree, Science 266, 1003 (1994).

[16] R. A. Gray et al., Circulation 91, 2454 (1995).

[17] A. T. Winfree, The Geometry of Biological Time (SpringerVerlag, New York, 1980).

[18] Z. Qu, J. Weiss, and A. Garfinkel, Am. J. Physiol. 276, H269 (1999).

[19] V. S. Zykov, Simulation of Wave Processes in Excitable Media (Manchester University Press, Manchester, 1987).

[20] R. R. Aliev and A. V. Panfilov, Chaos, Solitons Fractals 7, 293 (1996).

[21] A. V. Panfilov, S. C. Müller, V. S. Zykov, and J. P. Keener, Phys. Rev. E 61, 4644 (2000).

[22] F. Fenton and A. Karma, Chaos 8, 20 (1998).

[23] S. Newhouse, D. Ruelle, and R. Takens, Commun. Math. Phys. 64, 35 (1978).

[24] I. Aranson and I. Mitkov, Phys. Rev. E 58, 4556 (1998).

[25] H. Henry and V. Hakim, Phys. Rev. E 65, 046235 (2002).

[26] Z. Qu, F. Xie, and A. Garfinkel, Phys. Rev. Lett. 83, 2668 (1999).

[27] M. Wellner, O. Berenfeld, and A. M. Pertsov, Phys. Rev. E 61, 1845 (2000). 